\title{
Exploring the Internet of Things: an Interdisciplinary Workshop Approach
}

\author{
Dr. Sarah Eagle \\ Graduate School of Education \\ University of Bristol \\ Helen Wodehouse Building \\ 35 Berkeley Square \\ Bristol BS8 1JA \\ s.eagle@bristol.ac.uk
}

\author{
Dr. Peter Bennett \\ Department of Computer Science \\ University of Bristol \\ Merchant Venturers Building \\ Woodland Road \\ Bristol BS8 1UB \\ peter.bennett@bristol.ac.uk
}

\begin{abstract}
This paper gives an account of our experience running a workshop series exploring the 'Internet of Things' which was designed to focus on the idea the Internet of Things may develop from interdisciplinary collaboration and hacking/tinkering/making as opposed to large-scale corporate development.
\end{abstract}

Internet of Things. Workshop. Design. Prototyping.

\section{INTRODUCTION}

This paper gives an account of the authors' experience of running a series of interdisciplinary workshops entitled "Exploring the Internet of Things, Creativity and Learning", held between September 2012 and June 2013. The workshop series was inspired by the possibility that innovation in the 'Internet of Things' (IOT) will arise from the creativity of interdisciplinary communities of people who come together to 'tinker' with objects and technology.

IoT refers to an emerging development whereby everyday objects can simply and cheaply be linked to the Internet, providing information about their identity, status, or location, and/or receiving information from external sources. Policymakers envisage large-scale developments: "an allpervasive omnipresent socio-economic fabric, made up of converged services, shared data and an advanced wireless and fixed infrastructure linking people and machines to provide advanced services to business and citizens" [1] and attention has been given to constraints on infrastructure and issues of data security. The emergence of the 'maker movement', which includes those who connect objects with the Internet to produce diverse and often playful inventions, has received less attention. The technologies that enable people to build 'smart' objects are increasingly easy to obtain and varieties of easy-to-use 'kit' are now coming onto the market. Across the world, hackspaces in which people "come and tinker" with open source hardware have sprung up, of which some 40 are in the UK.

Led by researchers from university departments of Education and Computer Science, the workshop brought together a group of people from a variety of academic and practical backgrounds and disciplines to participate in the activity of 'making', exploring, in an informal atmosphere, how 'things' can be connected to information and to other objects, and the implications of doing so. We anticipated that the people involved in the workshop would also begin to tinker in a more abstract sense, and that there might be some making in the form of ideas and research questions. We were interested in what might arise if we set up an environment that was conducive to imaginative activity and discussion amongst people who took differing theoretically informed perspectives on creativity, innovation, and future forms and uses of hardware and software.

Over the course of four workshops we achieved, amongst the group, a level of intellectual excitement, pleasure in making, and broad interdisciplinary discussions. Participants found the experience of sharing their everyday thoughts about technological 'making' and future directions for technology, stimulating and rewarding, and early reports suggest that a number of new collaborative projects have arisen between people who worked together during the project. In this paper we describe, from our own perspectives as an educational researcher and researcher in $\mathrm{HCl}$ respectively, the lessons learned and the suggestions we would make for further workshops. 


\section{WORKSHOP DESIGN}

In designing the workshop we were inspired by the 'Blackwell-Leach Process' interdisciplinary design, which has been successfully applied in 'sandpit' meetings. It advocates that facilitators establish a setting where clients create their own culture [2] and advises mixing people of disciplines and of seniority, attending to subtleties of hosting, to the value of preparatory work of establishing relationships with participants, and to the quality of the environment and refreshments provided - but also for facilitators themselves to demonstrate honesty of engagement, of critique, and of their own capabilities.

\subsection{Participants}

The task of setting up the workshop began several months in advance of the first meeting, by making contact with a variety of different groups including academic groups within the University of Bristol and with people from Bristol Hackspace [3]. The groups were introduced to the project and invited to raise their own points of interest. The following gives an example of the range of perspectives of people who were interested in the workshop, and who signed up to attend:

- A group of social geographers who were concerned with relations between people and the material world, and the spatial habits of thought that inform the ways in which these relations are imagined and practiced.

- Educational researchers who were interested in learning through 'doing' from an enactivist perspective [4] and also in applying Andy Clark's ideas about how technology can enhance us as humans [5].

- A researcher from the Systems Centre, Faculty of Engineering, who was interested in how loT could create greater opportunities for userdriven urban infrastructure development.

- A researcher involved in information technology research and development to support the university was interested in thinking about loT as a potential future opportunity for using 'off the shelf' hardware and components to support researchers

- A teacher of student doctors was interested in the quality of conversations that can take place when people make things together, as was an educator of science communicators interested in the value of making for science education.

- A historian expressed his interest in the workshop as part of his interest in material culture and heritage.

- A researcher from the university's department of Drama: Theatre, Film, Television described her interest as being in materiality and place, media and objects.
The workshop was also attended by academics from the Department of Computer Science and the Faculty of Engineering, and by members of Bristol Hackspace who were familiar with Arduino, interested in loT and pleased to share their skills and interests with academics.

One aspect we felt was important was that the workshop was run neither by technologists nor artists, but by cross-disciplinary creative technologists with experience across art and technology. We were fortunate that Bristol is home to a flourishing community of animators and puppeteers, some of whom combine skills in robotics with puppetry, and invited members of the group Rusty Squid [6] to help facilitate the workshop.

\subsection{Workshops}

Workshops typically lasted for four hours, beginning or ending with a buffet lunch to allow time for informal conversation. For the first workshop, participants were assigned into groups of four on the basis of information they had given in advance, mixing academic disciplines and backgrounds and arranging that each group had one member with considerable experience of programming software. On the basis that hearing what others are saying can trigger ideas and discussion, seating was organised so that people had to sit close to one another, two groups sharing one large table.

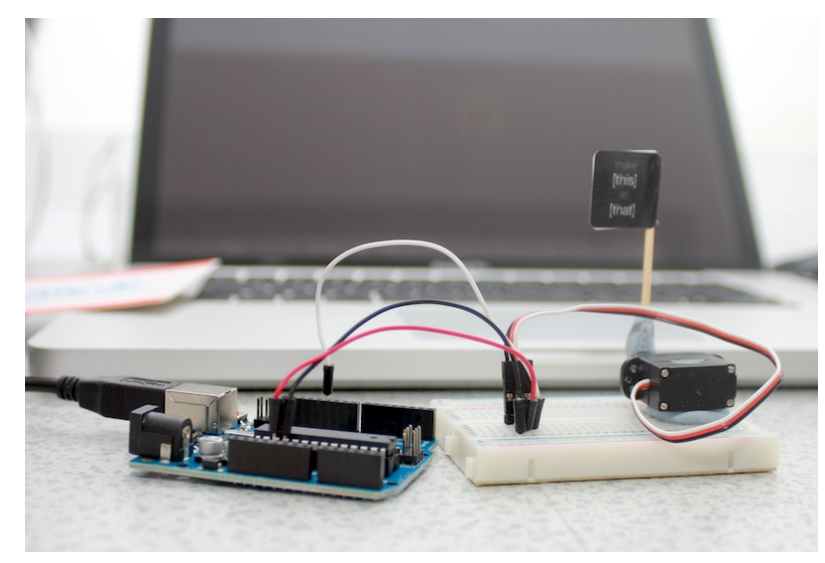

Figure 1 Twitter flag used as an example of a physical action caused by activity on the web.

The first workshop was designed as an introduction to IoT, and began with an outline of the concept, including video that promoted the potential of internet connected objects, chosen to illustrate visions of efficiency on the one hand [7] and playfulness on the other [8]. A Creative Technologist introduced the potential for connecting objects with data for some arts projects involving robotics and connectivity (Fig.1). The main activity for the workshop was hands on activity with the Arduino and prototyping materials. 
Arduino was introduced as a means that had made connecting objects with data an accessible activity, for hobbyists and professionals alike. Small groups, each of which included at least one person of a technological background, were given Arduino, paper, card, foam, pens, etc, invited to talk, make and play as they chose. The Creative Technologist offered support and inspiration to each group during the course of the afternoon. Each group finally presented their outcomes - which were in some cases a description of the fruitful discussions in their group and in others demonstrations of their prototype (see Outcomes below).

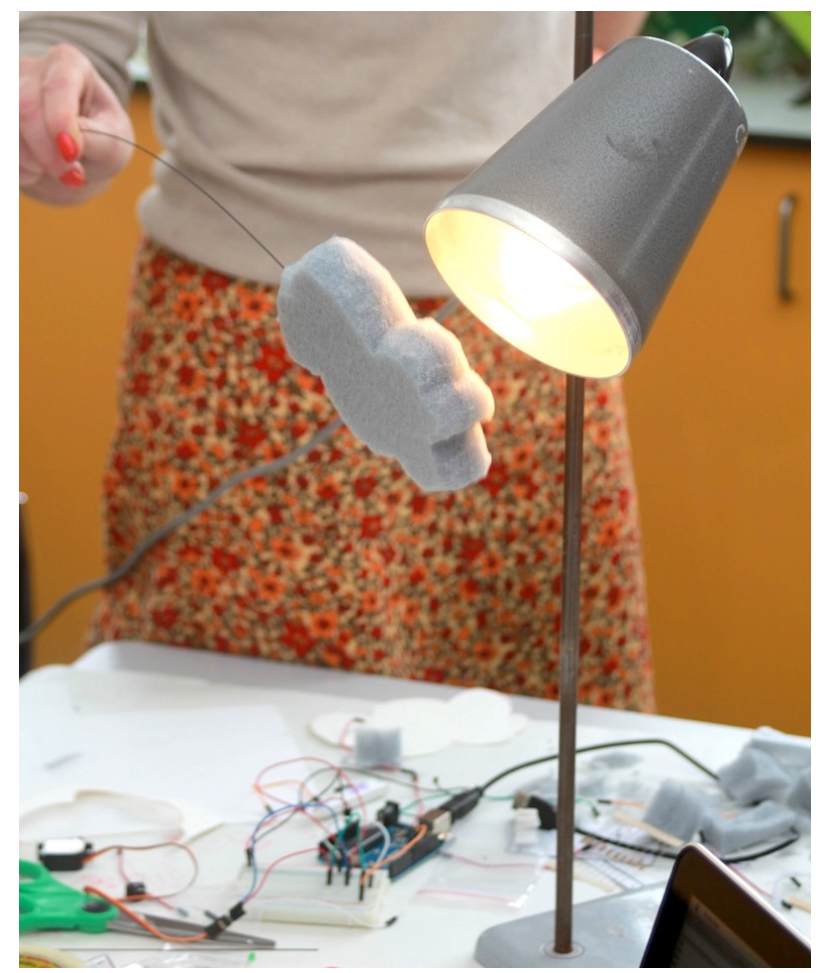

Figure 2 Simulating a cloud for a weather monitoring station.

In the second workshop we provided aruduino and prototyping materials as before, anticipating that groups would pick up where they had left off before. However, the group of twelve people at this meeting enthusiastically engaged in full-group discussion that lasted for the full duration of the workshop. The discussion centred on social technical and artistic conceptions of loT, and no physical 'making' took place, which was regretted by some of the participants.

In the third workshop, we introduced a simple task: three groups, again working in close physical proximity, were asked to make paper fortune tellers and to see what ideas and discussions arose. This task was designed to address the problem with the lack of making in the previous workshop whilst still encouraging open-ended conversation. Audio recordings were made throughout the workshop to capture the flow of conversation. An flavour of some of the ideas that emerged and discussions that ensued is given below.

\section{OUTCOMES}

We perceived three categories of outcome: rich discussion, creative activity, and the production of theoretically inspired written material.

\subsection{Group discussion}

A number of themes emerged during the series of workshops. Many of the ideas interweave, and the discussion took part across the different workshop sessions. Here we represent some of the recurring concepts, indicating the extent to which we felt the conversations had been able to develop:

Privacy \& ownership. Connecting personal objects to both other objects and the Internet poses a number of privacy issues that where frequently raised. Beyond these issues comes the question of who owns the data, and whether the conversations between objects are stored anywhere for long-term use.

Relationship between the Internet of Things, the Internet and the Web. During the workshop we were playing with very small, localised networks that didn't connect to the Internet. Are 'things' accessible via the web? Do networks of objects connect to the Internet, or remain separate?

Topology of Things. There were clear trends developing during the workshop discussion about how things communicated. The first step was a series of playful explorations of one-to-one communication between a person and an object. The next step, taken by some groups, was a discussion of many-to-one, or one-to-many communication. Within the context of the workshop, the final step (many-to-many communications) did not gain as much attention.

Language of Things. A common assumption was that the loT will allow any object to talk to any other object. Those with technological knowledge were able to contribute the idea that groups of objects may have different languages that are either completely or partially comprehensible by each class of objects. This then led to interdisciplinary conversations about the possible development of geographically distinct dialects that may evolve as different local communities develop their own loT culture, and the implications if this was to happen.

Inclusion of old objects. One theme for discussion was whether existing objects be physically hacked to connect to loT? This opens up the possibility of adding almost anything to the loT via some careful hacking. The benefit of old objects includes pre-existing emotional attachment and the potentially more environmentally friendly option of 
'upgrading' objects rather than binning them in favour of off-the-shelf loT enabled objects.

Learning as coming to know. Another theme was learning in the course of doing: with Arduino, learning through following stages of instructions, beginning by making a light blink and moving beyond. The physical detail of Arduino, its components, and the software interface were considered, asking what is made explicit (and attended to by the user) and what is implicit and 'becomes' part of what is known in the course of engagement; for example, the new 'maker' can attend to conceptual ideas about linking data and activity in an object, or to the detail of how current is flowing between breadboard and Arduino.

\subsection{Creativity}

Our intention had been that the workshop would begin with hands on activity, giving those who attended an opportunity to experience 'making' as connecting 'things' to information and to other objects, and to move from this to exploring implications. There was discussion of the value of 'making' as establishing a context for discussion of concepts and ideas in science and technology, and of the creativity stimulated when people of different disciplines 'play' together. We illustrate the last point with extracts from group activity in the third workshop:

Group 1 began with the idea of automating the folding of paper to make a 'fortune teller'

"It's a series of flat surfaces. With servos we could fold it into quarters, turn it over, fold it in again."

"But the thing is with it's the pleasure, it's the actual movement that's the real pleasure. Would we take the pleasure away from people by automating it?"

In a verbal account of their work, the group said:

\begin{abstract}
"We were playing with movement more than fortune telling. We are thinking about time, and what happens if you were keeping it open for a certain amount of time, and then a different time. And then we thought, to illustrate that, as using it as a musical device. So, we had different frequencies for different orientations. So if that is $\mathrm{x}$, that's that one frequency and that orientation is another. So, with two circuits, then how can we change the frequency using time, as well. So for example, one second here, and two seconds here, that's a certain frequency" (demonstrates fortune teller as input to laptop running MaxMSP programme)
\end{abstract}

Group 2 blended the idea of the fortune teller as a device for selecting from a series of unknown options and the idea of creating plots and content for a film:
"You could have a fortune teller and its eye is a webcam, it could be a magic wand, and it comes up with your fortune"

"There's counting or spelling ritual for coming to the a choice of which flap to open. We can put a, fortune, a set of instructions under the flaps"

"It could be a film making different plots"

"The instructions will tell the kids that they have to choose videos, the idea behind it is that children have to make something and connect it to the internet somehow, and they might have complete control over what they put, moving image and a bit of paper. All with smartphones, or in front of a computer with a webcam."

Group 3 did not move into prototyping, but spent the time discussing different ways of linking the paper fortune teller to data, through physical contact between closing flaps, to representing data

\begin{abstract}
"The two movements are orthogonal, it's the whole fundamental of communication. You could have three states - one this way, none or one that way. You could link it with the Arduino. Two, one, three states. Morse code? DTMF? 4 by 3 , so seven unique tones, harmonically not related to one another. Even with a poor signal you can still detect which - you could do morse code, couldn't you extend - one could be dot and one dash"
\end{abstract}

These three examples illustrate two distinct patterns of working. Groups $1 \& 2$ began by playing with the material objects; Group 3 preferred to work methodically, feeding back that they wanted to accumulate a deep understanding and only then plan what they would create:

\begin{abstract}
"It is nice to play with things, but part of says, you need to plan what you are making before you start work. We need to be clear about the resources we have. The capacity we have to do things relies on that.
\end{abstract}

In the first workshop the latter approach was characterised by groups who worked through a series of exercises designed to familiarise users with Arduino kits. Other groups worked through the first, 'hello world' exercise, then went straight on to inventing. For example, one group examined a servo, considering how its movement might be used to animate a paper structure. They then explored the varieties of movement they could obtain from the servo, discussing the possibilities for connecting to different data that would trigger different movements in their structure.

Whether working playfully or methodically, audio recordings reveal that the interdisciplinarity of groups contributed to considerable diversity in contributions to discussion, and this seemed to stimulate further generation of ideas and interest. 


\subsection{Written Output}

When planning the workshop, particularly in recognition of the range of perspectives that participants were bringing, we had envisaged that we would move from making in a physical sense to making in an academic sense, producing collaboratively written papers that explored aspects of loT from multiple perspectives. During the second workshop we carried out a collaborative writing task, and set up an online group, but found that only a few people accessed and contributed to documents and that discussion was sporadic. Some reasons for this are suggested in the section below.

\section{REFLECTIONS}

Given that the aim of the workshop was exploratory, no specific outcome was expected, although it was anticipated that interdisciplinary working would be stimulated and research partnerships might develop. It was clear from the high return attendance that people found the conversations and activity very rich and rewarding, and feedback was that participants felt that their own work was stimulated by an opportunity to discuss their everyday ideas with people who thought and worked in very different ways, but were also fascinated by the subject matter. The following list of problems, therefore, is from the perspective of the disciplinary allegiance of the two authors and expresses, on the one hand, our perceived difficulties in handling the complexity of the concept of loT in the workshop, and, on the other, our perceived difficulties in orchestrating theoreticallyinformed academic explorations of the subject matter.

The concept of the Internet of Things: the workshops were not designed to 'teach' about loT nor to come up with prototypes; groups were given a starting point (aruduino, fortune teller) and invited to collaborate as they pleased, in the course of engaging with a range of prototyping materials. However, we felt that there had been some difficulty in arriving at anything but a fairly simplistic conceptual model of IoT, for the following underlying reasons:

- Scalability. The Internet of Things will potentially operate on a massive scale. The problem encountered during the workshops was how to scale up our explorations of single interactions, up to a scale where millions of object may be simultaneously interacting. Storyboarding rather than physically prototyping may encourage this large-scale mode of thinking.

- Network Layer. Prototyping a network protocol is perhaps too time-consuming for a one-day workshop. However this network level structure is a key area of research. Problems were encountered in discussing this more technical aspect of the loT and opening up this area for debate amongst the less technologically minded in the group. One method we attempted was to shift the conversation from network protocols to 'the language of things' which allowed a higher level debate to take place around the lower level technical complications of connecting many different types of object to the loT.

- Miniaturisation. Many of the proposed objects for connecting to the IOT where rather small, posing the problem of how relatively large hardware such as Arduinos and servo-motors could be integrated with them. This raises the question of how miniature electronics can be integrated into loT objects whilst remaining accessible and hackable by the hobbyist.

- Programmability. A major problem we encountered in the workshop was how to program the communication and behaviour of the networked objects. There is the initial technical problem of many levels of software knowledge needed from low-level hardware interaction through to network protocols and web programming. And there is there is also the problem of how the objects can be programmed by the owner of the object. Possible solutions for programming the loT ranged from the use of simple scripting languages, through to zoomorphic behavioural training of networked object, slowly sculpting the objects behaviour through positive and negative reinforcement.

\section{Embedding academic writing within workshop} sessions. We noted the written material produced is less rich than the transcripts of discussion, and more difficult to facilitate. One explanation for this is offered by the participants themselves: the majority of those present during the writing session expressed their preference for making and communicating verbally and said they found writing was a longer, more drawn-out process, with just two participants saying that writing was the way that they preferred to work. We also recognise that may academics feel overstretched and are less likely to write collaboratively unless working towards some goal (e.g. grant application, academic paper).

\section{IMPLICATIONS}

The mix of physical making, writing software, hacking hardware and theoretical thinking takes a lot of time, especially in the time taken for the group to switch between modes of working. We found that making the deliberate (but difficult) shift from hands-on work to thinking about theory is necessary for the conversation to progress. Further 
workshops perhaps run a number of different groups in parallel, each working on a different aspect at the same time, with a deliberate rotation of people between then groups in order to propagate ideas. We feel that this mixing of groups is important, as many participants were drawn to the workshop by the opportunity for hands-on hacking, and would be put off attending by spending the whole time working on written work or theory.

The balance between broad and detailed methods of thinking was hard to achieve. We found that concentration on one design (such as the fortune tellers) reduced the size of the design space sufficiently that cycling between broad modes of thought to technical details became manageable. The difficulty of this working method is that the focus of the workshop is then limited to the one design, so the choice of which design to focus on becomes a rather contentious issue. Perhaps stressing that the design focus such as the fortune teller is only a stand-in object on which to focus the discussion would help enable participants who are not used to this method to ease into this way of working.

We found that the pre-workshop preparation of establishing a broad group of participants and generating a perception of informality from the outset was a very important part of the workshop process. We suggest that progression might be facilitated by further preparatory work, perhaps through one-to-one conversations where IOT related concepts can be explored at the participant's own pace encouraging them to think about their disciplinary perspective and generate questions and ideas prior to the workshop.

The introduction of working electronics felt necessary for exploration of ideas, but it may have proved to be more of a hindrance to the formation of theories and concepts, as the time taken to achieve a working demo was prohibitively long compared to the speed in which ideas could be explored by discussion around sketches.

Although we wished to avoid being prescriptive, a clear definition of the nature of a collaborative outcome might help participants focus on generating and presenting ideas. Such an outcome could be a website or blog including images, text and video which could be updated throughout the sessions. Another possibility would be the creation of a number of different scenarios of the loT encapsulated in a number of 'design fiction' stories. A third possibility could be a 'library of conversational objects', in essence a small exhibition perhaps contained on a bookshelf that could be placed in a public setting such as a library and used to spark debate around the topic.
Ultimately the aim of the workshop series was to help establish connections between various groups that may not have had a platform to meet. As such developing methods for maintaining these wide ranging conversations and providing a forum for continued work outside of the workshops is a key area for further work. A suggestion that has already had some support is that we move the discussion from a workshop setting in to a more informal group, who could continue to meet and develop ideas. For further interdisciplinary workshops, would hope to use the lessons learned to plan and deliver a longer series of events, for which 'informal creativity' and well-resourced hospitality is part of the attraction that keeps people coming back together.

\section{CONCLUSION}

We were successful in bringing together academics of different disciplines with creative and hobbyist technologists to hold rich and stimulating discussions in an atmosphere that was designed to stimulate bringing creativity and technology together. However, as a concept, the Internet of Things is a difficult topic to workshop since the topic is such a broad one, with a level of detail required for engagement at each layer technological, social, political, and creative.

\section{REFERENCES}

1 Townsend, E. (2011, May) UK Future Internet

2 Blackwell, A.F., (2006) Designing knowledge: An interdisciplinary experiment in research infrastructure for shared description. Crucible Network for Research in Interdisciplinary Design, University of Cambridge

3 Bristol Hackspace http://bristol.hackspace.org.uk

4 Varela, F., Thompson, E., \& Rosch, E. (1991). The embodied mind: cognitive science and human experience. Massachusetts: The MIT Press.

5 Clark, A. (2003). Natural-Born Cyborgs: Minds, Technologies, and the Future of Human Intelligence. Oxford University Press.

6 Rusty Squid http://rustysquid.org.uk/

7 SmartThings www.smartthings.com

8 Makey Makey www.makeymakey.com 